\title{
Semantic priming and retrieval from lexical memory: Evidence for facilitatory and inhibitory processes
}

\author{
JAMES H. NEELY \\ Yale University, New Haven, Connecticut 06520
}

\begin{abstract}
Immediately prior to each visually presented target letter string to which the subject made a speeded word-nonword classification response, a visually presented prime to which no overt response was required was shown for 360,600 , or 2,000 msec. For word (W) target trials, the priming event was either a semantically neutral warning signal (Condition NX), a word semantically related to the target word (Condition R), or a word semantically unrelated to the target word (Condition U); for nonword (N) target trials, the priming event was either the neutral warning signal (Condition NX) or a word prime (Condition WP). For the $W$ target trials, reaction times (RTs) were slower in Condition $U$ than in Condition NX and equally so for all three prime durations; RTs were faster in Condition $R$ than in Condition NX and to a greater degree for the 600 - and 2,000-msec prime durations than for the 360 -msec prime duration. For the N targets, RTs were faster in Condition WP than in Condition NX and equally so for all prime durations. These results were interpreted within the framework of a two-factor theory of attention proposed by Posner and Snyder (1975a).
\end{abstract}

In the lexical decision task, a subject must decide as quickly as possible whether a visually presented letter string is a common English word or nonword. A commonly obtained finding (e.g., Meyer \& Schvaneveldt, 1971; Meyer, Schvaneveldt, \& Ruddy, Note 1) is that subjects are quicker to respond that a target letter string (e.g., NURSE) is a word when the immediately prior target was a semantically related word (i.e., DOCTOR) than when the immediately prior target was a semantically unrelated word (e.g., BREAD). Schvaneveldt and Meyer (1973) have proposed two models, each of which can account for this semantic-facilitation effect. Both models share the assumption that, in terms of a spatial metaphor, the logogens for semantically related words are located nearer each other in semantic memory than are the logogens for semantically unrelated words (cf. Morton, 1970). According to the spreading excitation model, when a stimulus word activates its logogen, this activation spreads to adjacent, semantically related logogens but not to remote, semantically unrelated logogens. Thus, if a word that is to be processed is presented before there has been a complete decay of the logogen activation produced by a previously presented semantically related word, the activation level in the logogen of the to-be-processed word will initially be higher (due to the activation that has spread to it) than it would

This research was supported by Grant $40310 \mathrm{X}$ to Dr. Michael I. Posner from the National Science Foundation and was conducted while Dr. Posner was a Visiting Professor at Yale and while the author was a National Science Foundation predoctoral fellow. I am indebted to Dr. Robert G. Crowder, Dr. Alice F. Healy, and Dr. Posner for several interesting discussions about this research. Requests for reprints should be sent to the author, who is now at the Department of Psychology, University of South Carolina, Columbia, South Carolina 29208. have been had it been preceded by a semantically unrelated word; the result will be a facilitation in its processing. According to the location shifting model, a limitedcapacity attentional mechanism can "read out" the information stored at only those logogens upon which it is focused, and it must therefore be shifted before the information stored at an unattended logogen can be analyzed in preparation for response initiation. Thus, in terms of the location shifting model, the semanticfacilitation effect occurs because attention "traverses a shorter distance" when it shifts between semantically related logogens than when it shifts between semantically unrelated logogens.

Meyer, Schvaneveldt, and Ruddy (Note 1) conducted a lexical decision experiment designed to distinguish between these two models. Within each triplet of successively presented items, an item could be a word semantically related to another word in the triplet ( $R$ items), a word semantically unrelated to other words in the triplet (U items), or a nonword ( $\mathrm{N}$ items). The spreading excitation model predicts that the reaction times (RTs) to the second R item in a RUR triplet should be faster than RTs to the comparable item in the UUU control triplet-to the degree that the excitation spreading from the first $\mathrm{R}$ word's logogen has not dissipated during the time required for the response to the intervening $U$ word. On the other hand, the location shifting model predicts that the RTs to the second R item of the RUR triplet should never be faster than the RTs to the comparable item in the UUU control triplet, since in both the RUR and UUU triplets the necessary shift in attention between the second and third items would always be between semantically unrelated items. The results were that RTs to the second $R$ item in the RUR 
triplets were faster than the RTs to the comparable item in the UUU triplets, thus supporting the spreading excitation model. Schvaneveldt and Meyer (1973) obtained similar results in a simultaneous presentation paradigm and concluded that the semantic-facilitation effect in the lexical decision task should be attributed to spreading activation rather than to location shifting.

Quite recently, Posner and Snyder (1975a) have taken exception to Schvaneveldt and Meyer's (1973) conclusion that a limited-capacity attentional mechanism is not involved in the semantic-facilitation effect. In their theorizing, rather than viewing spreading activation and limited-capacity attention as mutually exclusive processes, as Schvaneveldt and Meyer (1973) have viewed them, Posner and Snyder (1975a) have assumed that spreading activation and limited-capacity attention can operate in conjunction with each other to modulate performance. Thus, from Posner and Snyder's theoretical perspective, although the Meyer et al. (Note 1) and the Schvaneveldt and Meyer (1973) data did indeed provide evidence for spreading activation, they did not rule out a contribution of the limited-capacity attentional mechanism to the semantic-facilitation effect obtained in the lexical decision task. In fact, since a decay of spreading activation was not sufficient to explain the complete loss of facilitation that was obtained for RNR triplets in the Meyer et al. experiment, Posner and Snyder argued that the Meyer et al. results actually suggested that the semantic-facilitation effect in the lexical decision task is due to the operation of both a limited-capacity attentional mechanism and a spreading activation process.

The purpose of the present experiment was to examine further the roles of limited-capacity attention and spreading activation in the semantic-facilitation effect in the lexical decision task. A crucial feature of the design of the present experiment is that, following the recommendation of Posner and Snyder (1975a), the target word to which the subject was to make a lexical decision was preceded by a-semantically neutral warning prime consisting of a series of Xs (Condition NX) on some trials and by either a semantically related word prime (Condition $\mathrm{R}$ ) or a semantically unrelated word prime (Condition $U$ ) on other trials. The importance of including an NX warning prime which does not direct the limited-capacity attentional mechanism to a specific set of word logogens nor activate, via spreading activation, the logogen for the target word is that it provides a baseline for assessing the limited-capacity attentional effects and the spreading activation effects produced by the $U$ and $R$ word primes, with their warning signal effects (cf. Posner \& Boies, 1971) partialled out. In terms of the cost-benefit nomenclature adopted by Posner and Snyder (1975b), if RTs to the target letter string are faster when the target follows a word prime than when it follows the NX prime, the word prime is said to have facilitated the processing of the target item; if, on the other hand, RTs to the target letter string are slower when the target follows a word prime than when it follows the NX prime, the word prime is said to have inhibited the processing of the target.

If the semantic-facilitation effect is replicated in the present experiment, subjects should be much faster in responding "word" to a word target in Condition $\mathrm{R}$ than in Condition U. More importantly, if, in comparison to Condition NX, subjects are slower to respond "word" to the word targets in Condition $U$, the limited-capacity attentional mechanism would be implicated in the semantic-facilitation effect because, in the PosnerSnyder theory, inhibition is produced by misdirected attention (due to its limited capacity) but is not produced by spreading activation. ${ }^{1}$ If, on the other hand, there are no differences in the RTs to the word targets in Conditions $N X$ and $U$, the semantic-facilitation effect could be solely attributed to spreading activation, as Schvaneveldt and Meyer (1973) have suggested. The stimulus onset asynchrony (SOA) between the prime and the target was also varied in the present experiment in order to chart the time course of the facilitation and inhibition effects (should they be obtained). The PosnerSnyder theory predicts that the facilitation in Condition $\mathrm{R}$ should build up faster than the inhibition in Condition $U$, because spreading-activation facilitation is fast and automatic, while inhibition depends on the slower and more deliberate commitment of conscious attention.

Another important feature of the design of the present experiment is that it uses words other than the target words themselves as primes to activate, via spreading activation in Condition $R$, the logogen for the target word. This eliminates the opportunity for the subject to respond to the target on the basis of a physical match or mismatch between the target and the priming word. This is important because the inhibition obtained in the Posner and Snyder (1975b) experiments could have been produced by such a physical-match strategy rather than by a general inhibition associated with misdirected attention, as predicted by the Posner-Snyder theory. Because of the evidence for a physical-match strategy obtained in their experiments, Posner and Snyder (1975a, b) acknowledged that their theory would be more strongly supported if an inhibition effect was obtained in an experiment in which the prime and the target were not physically identical. If, in the present experiment, an inhibition effect was obtained in Condition $U$ and this effect was to be attributed to a general inhibition effect associated with the operation of limited-capacity attention, the processing of the nonword targets should also be inhibited by the word primes. This prediction is based on the assumption that part of the limited capacity of the attentional mechanism is depleted by the decision to focus attention and the act of focusing attention (cf. LaBerge, 1973). Thus, independently of the location shifting 
metaphor, which may be inappropriate for $\mathrm{N}$-target trials (see, however, Meyer \& Schvaneveldt, 1971) and NX-prime trials, an inhibition effect is predicted for the $\mathrm{N}$ targets solely on the basis of the word prime's depleting more attentional capacity than the $X$ prime, because its presentation results in the subject's performing the capacity-consuming operation of focusing his attention on semantically related logogens. ${ }^{2}$

\section{METHOD}

\section{Design}

As noted above, the dependent variable was the time to decide whether or not a target letter string was an English word. On half of the trials the target letter string was a common English word (W) and on half of the trials, a nonword $(\mathrm{N})$. Immediately prior to the presentation of the target letter string, a prime was shown for 360,600 , or $2,000 \mathrm{msec}$. [Since SOA and prime duration were confounded, it should be noted that in Posner and Snyder's (1975b) Experiment 1 , the facilitation and inhibition effects depended only on SOA, not on prime duration.] The SOAs were chosen to be considerably longer than those used by Posner and Snyder (1975b) because it was thought that a substantial amount of time would be required for the subject to read a priming word selected from a large stimulus set, i.e., the English lexicon, and for the subject to use that priming word to direct his attention to semantically related logogens rather than focusing his attention on the logogen for the priming word itself. (See Footnote 2.) As defined above, there were three kinds of primes for the $W$ targets: $R, U$, and NX. Since the $R$ and $U$ distinction was a pseudodistinction for the $\mathrm{N}$ targets, data from all word-prime $\mathrm{N}$-target trials were collapsed into a single word-prime (WP) condition. In short, for the $\mathrm{W}$ targets the complete design was a 3 (prime duration) by 3 (prime type) design and, for the $\mathrm{N}$ targets, it was a 3 (prime duration) by 2 (prime type, WP vs. NX) design, with each subject receiving each of the nine $W$ conditions and each of the six $\mathrm{N}$ conditions.

\section{Stimulus Materials and List Construction}

The priming words and the target words for the $\mathrm{R}-\mathrm{W}$ conditions were chosen from an atlas of normative free association data (Shapiro \& Palermo, 1968) such that, when the priming word served as a stimulus, the target word was given as the primary associate at least $40 \%$ of the time. For the U-W conditions, the priming words were reshuffled so that they did not appear on the same trial as did their primary responses. For the WP-N conditions, the priming words were chosen from the Kucera and Francis (1967) norms, so that they were matched on frequency with the priming words for the R-W and U-W conditions. The $\mathrm{N}$ targets were constructed from words drawn from the set of remaining words in the Shapiro and Palermo norms by changing one letter in each of the words (e.g., BRUSH to GRUSH and CREDIT to CREMIT). James (1975) has argued that, when pronounceable nonword targets such as these are used, the subject cannot classify words and nonwords on the basis of structural differences and must, therefore, "look up" the meaning of the word targets in order to make his lexical decision.

A base list consisting of six blocks of 42 trials was formed using these materials. The $\mathrm{W}$ targets for the first three blocks of 126 total trials were chosen from the 126 response terms taken from the associative norms. Since there were only $63 \mathrm{~N}$ targets from which to choose, a W or N target was "randomly" chosen such that in the first three blocks there were $63 \mathrm{~W}$ targets and $63 \mathrm{~N}$ targets, with no repetitions of a W or $\mathrm{N}$ target. The remaining $63 \mathrm{~W}$ targets appeared in the second three blocks and the $63 \mathrm{~N}$ targets used in the first three blocks were used once again. Thus, each $\mathrm{N}$ target occurred twice in the whole list, once in the first three blocks and once in the second three blocks, but no word appeared twice in the experiment, either as a target or as a prime. The priming conditions were randomly assigned to the targets, with the constraint that within each block of 42 trials there were 7 instances each of the $\mathrm{R}-\mathrm{W}, \mathrm{U}-\mathrm{W}, \mathrm{NX}-\mathrm{W}$, and NX-N conditions and 14 instances of the WP-N condition. Two other base lists were derived from the first base list by reassigning primes, so that across the three lists each $W$ target occurred in all three priming conditions and each $\mathrm{N}$ target occurred in both the WP and the NX conditions. The order of presentation of the targets remained the same for the three base lists. Three additional lists (for a total of six base lists) were constructed from the three base lists by interchanging the tcrgets in the first three blocks with those targets in the same serial position in the second three blocks.

The SOAs were blocked such that, in the first three blocks, the SOA was $360 \mathrm{msec}$ in one block, $600 \mathrm{msec}$ in another block, and $2,000 \mathrm{msec}$ in the remaining block, and likewise for the second three blocks. A base order of SOAs was developed by "randomly" assigning SOAs to blocks such that no two successive blocks were assigned the same SOA. Two other SOA orders were derived from it such that, across the three SOA orders, each of the six blocks was assigned to each of the SOAs.

Each of the 18 lists ( 6 base lists $\times 3$ SOA orders) was preceded by an identical practice block of 28 trials with $14 \mathrm{~W}$ targets and $14 \mathrm{~N}$ targets. There were no instances of the R-W condition in the practice list. Furthermore, the SOA was always $2,000 \mathrm{msec}$ for the first block of 14 trials and $360 \mathrm{msec}$ for the second block of 14 trials. Although there were no 600 msec SOAs in the practice list, subjects were told that they would receive three different SOAs in the experiment but that they would be given only the long and short SOAs in the practice block.

Each of the six blocks of 42 trials in the test sequence was preceded by two practice trials with no instances of Condition R-W. The two practice trials preceding each block were different for each block but identical for all 18 lists.

\section{Procedure}

Eighteen members of the Yale community were paid $\$ 2$ each for their participation in the 1-h session. All subjects were native English speakers and each subject was tested individually. Each subject was read general instructions describing the task and was told that the word targetswere common English words, so that the experiment would not be a vocabulary or spelling test. The subject was told to make fewer than $10 \%$ errors and to fixate his vision on both the priming slide and the target slide as long as it remained displayed. The subject was also told that he should try to avoid anticipating if a trial was going to contain a word or nonword target, since this was randomly determined, and that the experimenter would read out the RT for each trial so the subject could try to improve his times.

Each trial consisted of two slides, the priming slide and the target slide, successively rear projected on translucent Mylar near the center of a $12.0 \times 16.0 \mathrm{~cm}$ aperture. The priming event and the target event occurred in identical positions in the aperture. The offset of the priming slide was followed immediately by the onset of the target slide, which remained exposed until the subject responded by pressing one of two keys. The subject began each trial with the index finger of each hand resting on its corresponding key. For half of the subjects, the "word" response was assigned to the dominant hand and the "nonword" response to the nondominant hand; for the other half of the subjects, the opposite assignment was made. During the 9-sec intertrial inter$\mathrm{val}$, subjects were informed of their RT for the previous trial and were told whether or not they had made an error. The prime durations were controlled by Lafayette shutters and BRS digibit 


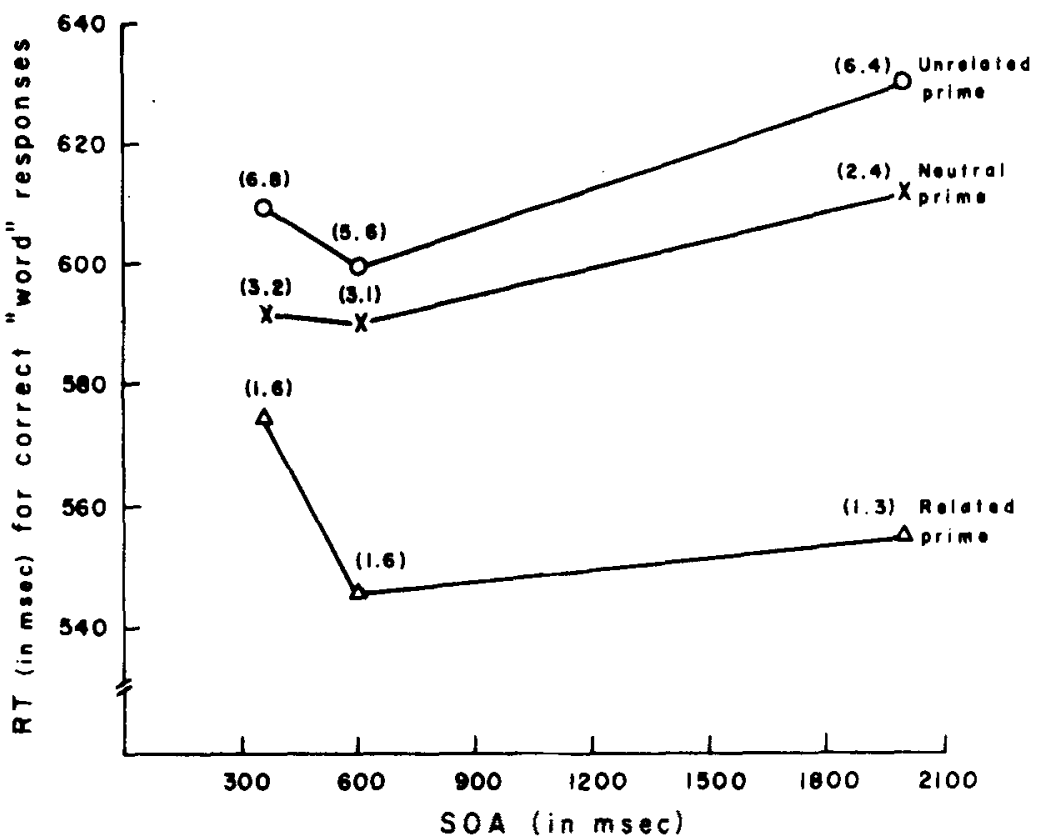

Figure 1. Reaction times (RTs) (in milliseconds) for correct responses in the wordtarget conditions as a function of stimulus onset asynchrony (SOA). Mean percentage errors are given in parentheses.

solid state timers; RTs were measured to the nearest millisecond by a Hunter Model 1520 timer.

After the instructions were read, the 28 practice trials were given. There was a 30 -sec intertrial interval between the 14 th and 15 th practice trials to allow the experimenter to change the SOA from 2,000 to $360 \mathrm{msec}$. After the practice list, the slide trays and the SOA were changed. This required about $2 \mathrm{~min}$ and then the first block began. All six blocks were separated by this 2 -min change-over period and, before each block began, the subject was told whether the SOA for that block would be the long, medium, or short time interval.

\section{RESULTS}

\section{Word Target Data}

Figure 1 displays the mean RTs for correct responses to the $\mathrm{W}$ targets for the different prime-type conditions as a function of SOA, with the mean percentage of errors given in parentheses. The number of observations upon which each of these means was based varied between 233 and 245 . The most salient aspect of the W-target data is that a large semantic-facilitation effect was obtained, i.e., RTs in Condition $R$ were, on the average, $54 \mathrm{msec}$ faster than RTs in Condition U. More importantly, this semanticsfacilitation effect was attributable to an average inhibition effect of $16 \mathrm{msec}$ in Condition $U$ (as compared to Condition NX) and an average facilitation effect of $38 \mathrm{msec}$ in Condition R (as compared to Condition NX). The inhibition effect seen in Condition $U$ remained relatively constant as a function of SOA, while the facilitation effect seen in Condition $\mathrm{R}$ increased from $17 \mathrm{msec}$ for the $360-\mathrm{msec}$ SOA to $56 \mathrm{msec}$ for the $2,000-\mathrm{msec}$ SOA. Another point of interest is that, for all three priming conditions, RTs tended to be fastest at the $600-\mathrm{msec}$ SOA, a result that is in accord with the finding of Posner and Boies (1971)' that a warning signal speeds up responses most at SOAs near 500 msec.

These conclusions were generally supported by a 2 (half of session) by 3 (prime type) by 3 (SOA) by 18 (subjects) analysis of variance. There was a highly significant effect of prime type $[F(2,32)=62.1, p<.001$, $\mathrm{MSe}=1,318 \mathrm{]}$. Subsequent $\mathrm{t}$ tests, using the appropriate error term from the overall analysis of variance as the error estimate, indicated that there was a statistically significant inhibition effect in Condition $U[t(32)=3.14$, $\mathrm{p}<.01]$ and a statistically significant facilitation effect in Condition $R[t(32)=7.69, p<.001]$. Fourteen subjects showed an inhibition effect for Condition $U$, and all 18 subjects demonstrated a facilitation effect in Condition R. Although the effect of SOA was not statistically reliable $[\mathrm{F}(2,32)=2.36$, MSe $=4,863]$, the interaction of SOA by Prime Type did reach conventional levels of statistical reliability $[F(4,64)=2.97, p<.05$, MSe $=$ $1,709]$. The facilitation effect for Condition $R$ was smaller at the 360-msec SOA than at the $600-\mathrm{msec}$ and 2,000 -msec SOAs $[\mathrm{t}(64)=2.42, \mathrm{p}<.01 ; \mathrm{t}(64)=4.04$, $p<.001$, respectively]. The Session Half by Prime Type interaction yielded $F(2,32)=5.45, p<.01, \mathrm{MSe}=1,537$, with the facilitation effect for Condition $\mathrm{R}$ being larger in the second half of the session than in the first half ( 48 vs. $28 \mathrm{msec}$ ) $[\mathrm{t}(32)=2.68, \mathrm{p}<.01]$, and the inhibition effect for Condition $U$ being larger in the second half of the session than in the first half $(23 \mathrm{vs.} 8 \mathrm{msec})[\mathrm{t}(32)=$ $1.99, \mathrm{p}<.06]$.

Since error rates were so low ( $3.6 \%$ overall), they were not subjected to an analysis of variance. Thus, the only point that needs to be made concerning the error rates is that there was no evidence for a speed-accuracy tradeoff, since there were more errors $(6.3 \%)$ in Condition $U$ than in Condition NX (2.9\%) and fewest errors (1.5\%) in Condition R.

\section{Nonword Target Data}

Figure 2 displays the mean RTs for correct responses to the $\mathrm{N}$ targets for the two prime-type conditions as 


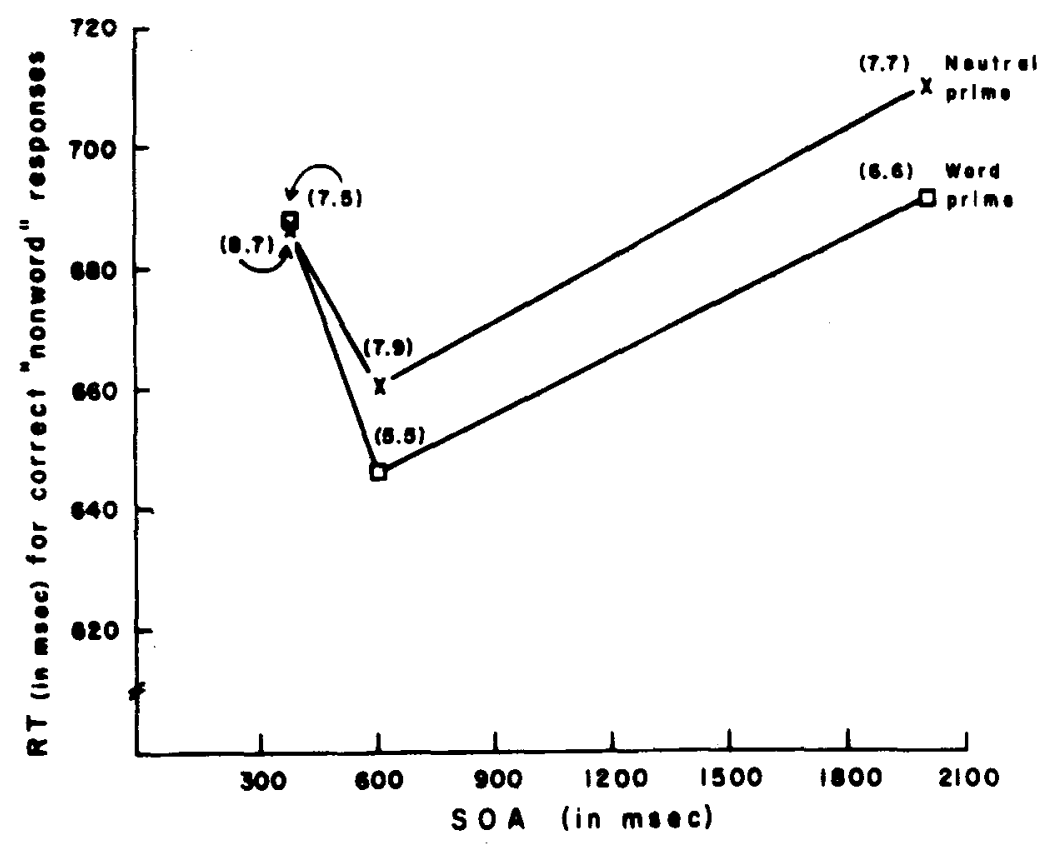

Figure 2. Reaction times (RTs) (in milliseconds) for correct responses in the nonword-target conditions as a function of stimulus onset asynchrony (SOA). Mean percentage errors are given in parentheses. a function of SOA, with the mean percentage of errors given in parentheses. The number of observations upon which each of the WP and NX means was based varied between 464 and 471 and between 230 and 232, respectively. There are two points of interest in the data in Figure 2. First, RTs in the WP condition were, on the average, $12 \mathrm{msec}$ faster than in the NX condition, and this facilitation effect increased with increasing SOAs. Second, RTs were fastest for both priming conditions at the $600-\mathrm{msec}$ SOA.

The statistical reliability of these effects was assessed with a 2 (half of session) by 2 (prime type) by 3 (SOA) by 18 (subjects) analysis of variance. The facilitation effect in Condition WP was statistically significant $[F(1,16)=6.43, p<.05, \mathrm{MSe}=1,200]$, with 14 of the 18 subjects showing the effect. However, the amount of facilitation produced by the word primes was not significantly greater for the 600 - and $2,000-\mathrm{msec}$ SOAs than for the 360-msec SOA $[\mathrm{F}(2,32)<1, \mathrm{MSe}=3,685]$, nor did the amount of facilitation differ for the two halves of the session $[F(1,16)=3.75, p>.05$, MSe $=1,546]$. The only other effect to reach conventional levels of statistical significance was the effect of $\operatorname{SOA}[F(2,32)=$ $6.96, \mathrm{p}<.01, \mathrm{MSe}=5,000]$. The RTs were faster at the $600-\mathrm{msec}$ SOA than at the $360-\mathrm{msec}$ SOA [t(32) = $1.87, \mathrm{p}<.05$, one-tailed] or the 2,000-msec SOA $[t(32)=2.55, p<.05]$. As noted above, this result is in accord with the findings of Posner and Boies (1971).

As with the word data, the error rates did not provide any evidence for a speed-accuracy tradeoff, since there were fewer errors $(6.5 \%)$ in Condition WP than in Condition NX (8.1\%).

\section{DISCUSSION}

The results of the present experiment, taken in conjunction with the results of Meyer et al. (Note 1) and Schvaneveldt and Meyer (1973), suggest that there may be two reasons why subjects are faster to respond that a target letter string is a word when it follows a semantically related priming word than when it follows a semantically unrelated priming word: (1) Activation spreads from the logogen for the priming word to the logogens for semantically related words, and (2) the subject uses the priming word to direct his limitedcapacity attention to logogens for words that are semantically related to the priming word. Within the framework of the Posner-Snyder (1975a) theory, evidence that the subject is using the prime to direct his attention comes from two sources. First, subjects were slower to respond that a target was a word when it followed a semantically unrelated priming word than when it followed a neutral warning signal. Such an effect would occur if the subject used the prime to direct his attention to semantically related logogens, since his attention would then be misdirected when the target was a semantically unrelated word. Second, the findings that the amount of inhibition seen in Condition $U$ and the amount of facilitation seen in Condition $R$ both increased with practice are congruent with the limitedcapacity attention interpretation because, as subjects receive more and more trials in which the prime and target are semantically related, they should be more likely to adopt the strategy of using the prime to direct their attention to semantically related logogens.

However, there are other data from the experiment that suggest that the inhibition effect obtained in Condition $\mathrm{U}$ may not have been due to misdirected attention. First of all, contrary to what the PosnerSnyder theory predicts, the inhibition effect in Condition $U$ did not increase as the SOA increased, but there may have been a failure to detect this predicted growth 
in inhibition because of its small magnitude, i.e., 15 msec. A possible reason why the inhibition effect was so small in Condition $U$ is that, unlike the previous lexical decision studies, the present experiment did not require that the subject respond to the priming word. (This was done in order to better control the SOA between the priming word and the target word and to equate the overt-response requirements for the neutral warning signal and the word primes.) Therefore, the subject may not have committed the limited-capacity attentional mechanism to the read out of information from the priming logogen, because the probability that a word prime was semantically related to the target word was only $50 \%$, conditionalized on the presentation of a priming word and a word target, and because the automatic facilitation effects may have been substantial enough by themselves, such that there was no overriding motivation for the subject to focus his attention on logogens semantically related to the priming word.

Notwithstanding this reasonable explanation for the small magnitude of the inhibition effect in Condition $U$, the problematic fact still remains that the inhibition effect was statistically significant and yet did not increase as the SOA increased. A proponent of the Posner-Snyder theory might maintain that, since the amount of inhibition did not increase with increases in the SOA, the small inhibition effect seen in Condition $U$ should not be attributed to the operation of the limited-capacity attentional mechanism. This argument can be made less tautological by an appeal to the nonword data. If the priming word was depleting some of the subject's limited-capacity conscious attention, according to the Posner-Snyder theory there should have been an inhibition effect for the $\mathrm{N}$ targets as well as for the semantically unrelated $W$ targets. However, RTs for the $\mathrm{N}$ targets were significantly faster in Condition WP than in Condition NX. Thus, within the framework of the Posner-Snyder theory, the facilitation effect for the nonword targets argues against the limited-capacity attentional explanation of the inhibition effect in Condition U. Equally important is the fact that the facilitation effect for the nonword targets also argues against other one-factor explanations for the inhibition effect obtained in Condition U. For example, it cannot be argued that the act of reading the priming word slowed down RTs in Condition $U$ because it should have had the same effect for the nonwords; nor can it be argued that subjects were slower to respond to the $\mathbf{N}$ targets in Condition NX than in Condition WP because they were less aroused following a "boring" $X$ prime than following a more interesting word prime, because the same arousal factor should have been operative for the $\mathrm{W}$ targets and should have produced a facilitation effect in Condition U.

Although the present experiment was designed to eliminate the physical-match strategy that Posner and Snyder (1975b) attributed to their subjects, the results of the present experiment nevertheless correspond to their findings. That is, in both the present experiment and Posner and Snyder's experiments, when the PosnerSnyder theory predicted an inhibition in the processing of a target array for which a positive response was correct, the predicted inhibition was obtained; however, when the Posner-Snyder theory predicted an inhibition in the processing of a target array for which a negative response was correct, the predicted inhibition was not obtained, but a statistically significant facilitation effect was obtained instead. The commonality in the results of these experiments suggests a possible explanation for the inhibition effect in Condition U.W and the facilitation effect in Condition WP-N. Although the design of the present experiment precluded a physical-match strategy, the use of highly stereotyped primary associates may have encouraged the subjects to adopt an associative matching strategy of generating the primary associate of the priming word, matching this self-generated item with the target item and then responding on the basis of a match or mismatch. For example, given CAT as a priming word, the subject would generate " $\mathrm{dog}$ " as a candidate for the target; if DOG was indeed the target, he would be fast to respond "word"; if any other letter string appeared as the target, he would have a tendency to respond "nonword." This tendency would speed up (and decrease errors for) a "nonword" response to TARK, but it would slow down (and increase errors for) a "word" response to NURSE. The idea that subjects adopted this strategy becomes more reasonable when one considers the structure of the present experiment. Since a W target was a word semantically unrelated to the word prime on half of the word-prime trials and a $\mathrm{W}$ target followed a word prime on only half of the word-prime trials, the subject could have based his response on an associative match, and he would have been correct and benefited on $75 \%$ of the word-prime trials (including $\mathrm{N}$-target trials).

of course, this associative-matching strategy does not explain the failure to find a statistically significant Inhibition by SOA interaction, because an associativematching strategy depends on a conscious attentional strategy of generating a word associatively related to the priming word; thus, it too should become more fully operative as the SOA increases. Nevertheless, the associative-matching explanation does serve to integrate the results of the Posner and Snyder (1975b) experiments with the present results. It also suggests that the use of a matching strategy is not confined to priming experiments in which the primes and targets are often physically identical, but rather may be used in all kinds of priming experiments, with the match being made at the level appropriate to the particular experiment. If future priming experiments should provide more evidence for some sort of matching strategy, the implication would be that in priming experiments consciousattention inhibition is not exerting its influence during the stimulus encoding or information read-out stage of processing, as is currently postulated by the Posner- 
Snyder (1975a) theory, but rather is having its effect in the response-decision stage of processing.

Whatever the ultimate theoretical resolution of the time course and the mechanism of the inhibition effects obtained in the present experiment may be, it would seem that the present experiment furnishes three empirical facts that should be counted among the core set of facts that any theory of semantic priming and the lexical decision process must accommodate if it is to be an adequate theory. The three facts are that, in comparison to a noninformative and semantically neutral warning-signal prime, a word prime (1) facilitates lexical decisions about a subsequently presented semantically related word, (2) inhibits lexical decisions about a subsequently presented semantically unrelated word, and (3) facilitates lexical decisions about a subsequently presented nonword.

\section{REFERENCE NOTE}

1. Meyer, D. E., Schvaneveldt, R. W., \&. Ruddy, M. G. Activation of lexical memory. Paper presented at the meeting of the Psychonomic Society, St. Louis, November 1972.

\section{REFERENCES}

Collins, A. M., \& Lofrus, E. F. A spreading-activation theory of semantic processing. Psychological Review, 1975, 82. 407-428.

JAMEs, C. T. The role of semantic information in lexical decisions. Joumal of Experimental Psychology: Human Perception and Performance, 1975, 1, 130-136.

Kučera, H., \& Francts, W. N. Computational analysis of present day American English. Providence, R.I: Brown University Press, 1967.

LABERGE. D. Identification of the time to switch attention: A test of a serial and parallel model of attention. In $S$.
Kornblum (Ed.), Attention and performance IV. New York: Academic Press, 1973.

Meyer, D. E., \& Schvaneveldt, R. W. Facilitation in recognizing pairs of words: Evidence of a dependence between retrieval operations. Journal of Experimental Psychology, 1971, 90, 227-234.

MorTon, J. A functional model for memory. In D. A. Norman (Ed.), Models of human memory. New York: Academic Press, 1970.

Posner, M. l., \& Boles, S. W. Components of attention. Psychological Review, 1971, 78, 391.408.

Posner, M. I., \& SNYDer, C. R. R. Attention and cognitive control. In R. L. Solso (Ed.), Information processing and cognition: The Loyola symposium. Hillsdale, N.J: Erlbaum, 1975. (a)

Posner, M. I., \& Swyder, C. R. R. Facilitation and inhibition in the processing of signals. In P. M. A. Rabbitt \& $S$. Dornic (Eds.), Attention and performance V. New York: Academic Press, 1975. (b)

Schvaneveldt, R. W., \& Meyer, D. E. Retrieval and comparison processes in semantic memory. In $S$. Kornblum (Ed.), Attention and performance IV. New York: Academic Press, 1973.

Shapiro, S. 1., \& Palermo, D. S. An atlas of normative free association data. Psychonomic Monograph Supplements, $1968,2,219-250$.

\section{NOTES}

1. Collins and Loftus $(1975$, p. 411) have made a similar assumption in their spreading-activation theory of semantic memory.

2. Since a priming word itself never appeared as a target and the subject was not required to respond overtly to the priming word, it is assumed that the subject would not continue to focus his attention on the logogen for the word prime itself, but would instead use the word prime to direct his attention to semantically related logogens.

(Received for publication January 8, 1976; revision accepted April 2, 1976.) 